\title{
Adrenal Hematoma Simulating Malignancy in Idiopathic Thrombocytopenic Purpura
}

\author{
Aleksandr A. Reznichenko
}

\begin{abstract}
Unilateral adrenal hemorrhages are unusual and have different etiologies. Very rarely adrenal hematoma could be presented as an adrenal mass and may have features of neoplastic lesion. There have been a number of case reports on adrenal hematoma that presented as an adrenal tumor in the past; however, there were no reports where it was described in patients with idiopathic thrombocytopenic purpura (ITP). We present a patient with ITP and large adrenal mass suspicious for malignancy, which was surgically excised, and turned out to be an adrenal hematoma.
\end{abstract}

Keywords: Adrenal hemorrhage; Adrenal hematoma; Adrenal mass; Idiopathic thrombocytopenic purpura; Adrenal carcinoma

\section{Introduction}

Adrenal hematomas occur rarely and have multiple causes [1]. They were reported in patients with trauma, long-term use of nonsteroidal anti-inflammatory drugs, neurofibromatosis, liver transplantation, primary and metastatic adrenal tumors, heparin-associated thrombocytopenia, anticoagulation, sepsis, and anti-phospholipid antibodies [2-4]. Adrenal hemorrhage could be spontaneous [2], and sometimes has clinical and radiographic features of adrenal neoplasm [2, 3, 5-9]. So far there were no reports of adrenal hematomas in patients with idiopathic thrombocytopenic purpura (ITP).

Adrenal masses require comprehensive workup to rule out hormone-producing tumor and malignancy. Lesions larger than $6 \mathrm{~cm}$ are rarely benign and should be excised [2]. We present a 65 -year-old female with ITP and $7 \times 9 \times 6 \mathrm{~cm}$ adrenal hematoma, simulating malignancy.

\section{Case Report}

A 65-year-old female with history of hyperlipidemia, hyper-

Manuscript accepted for publication August 19, 2015

Department of Surgery, Tulare Regional Medical Center, Tulare, CA, USA. Email: areznik9@yahoo.com

doi: http://dx.doi.org/10.14740/jcs278w tension, diabetes mellitus, hypothyroidism, coronary artery disease, depression, tonsillectomy, cholecystectomy, tubal ligation, colonoscopy, and arthroscopy presented with lower gastrointestinal bleed, thrombocytopenia, weakness, petechial rash and easy bruising. She was diagnosed with ITP and was treated with steroids. Her platelets count improved from 50 to 120 and she had colonoscopy with polypectomy. Three months later, she started to have abdominal pain in left upper quadrant (LUQ) radiating to her back. Abdominal computer tomography $(\mathrm{CT})$ showed $7 \times 9 \times 6 \mathrm{~cm}$ left adrenal mass with calcifications and central and peripheral enhancement with IV contrast, highly suspicious for primary adrenal carcinoma or metastatic disease (Fig. 1). Comprehensive endocrinology evaluation, including plasma and urine aldosterone, plasma renin activity, plasma and urine metanephrines, plasma and urine catecholamines, free cortisol and dehydroepiandrosterone (DHEAS) did not show hormonally active adrenal tumor. There was no evidence of adrenal insufficiency. Radiological evaluation with CT of the neck, chest, abdomen and pelvis was negative for any primary malignancy. Indication for surgery was large mass suspicious for primary left adrenal carcinoma. Patient underwent left adrenalectomy. Operation was started laparoscopically. During the procedure, significant desmoplastic reaction was noted around the mass with involvement of the splenic and left renal veins, and the case was converted to open. Final pathology evaluation of the excised mass showed

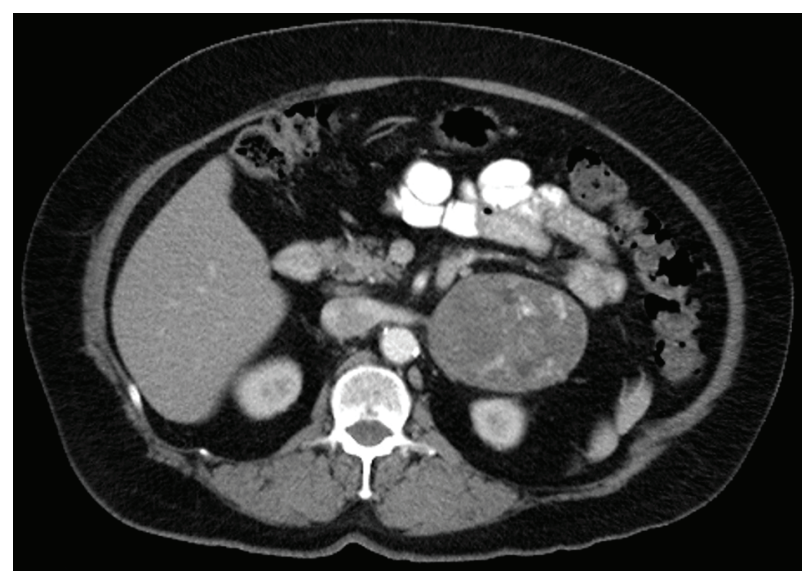

Figure 1. Abdominal CT with contrast, axial view. Left adrenal mass enhancing with IV contrast. 


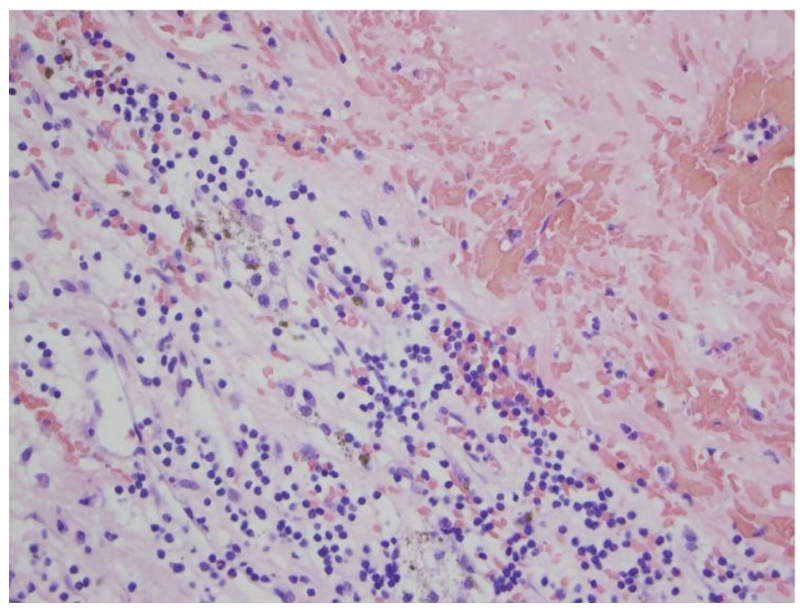

Figure 2. Pathology. Adrenal hemorrhage with necrosis.

organized adrenal hemorrhage with necrosis (Fig. 2). Patient had successful recovery and had no symptoms or recurrence for 2 years. Abdominal CT performed 24 months after surgery showed no evidence of recurrence (Fig. 3).

\section{Discussion}

Adrenal hematomas are rare entities and only few series were published [3, 4]. These lesions were most commonly described as a consequence of trauma, but also in patients taking non-steroidal anti-inflammatory drugs (NSAIDs), with neurofibromatosis, after liver transplantation, with primary and metastatic adrenal tumors, with heparin-associated thrombocytopenia, on anticoagulation, on hormonal therapy, with severe sepsis and anti-phospholipid antibodies [2-4]. Spontaneous idiopathic adrenal hematomas were also reported $[2,5,7,8]$.

Clinical presentation of adrenal hematomas varies, and may include flank or abdominal pain (like in our patient), fever, tachycardia, weakness, hypotension, nausea, vomiting, changes in mental status, weight loss, abdominal distention and palpable abdominal mass [2].

ITP is a clinical syndrome in which a decreased number of circulating platelets manifest as a bleeding tendency. The differential diagnosis includes such other causes of thrombocytopenia as leukemia, myelodysplasia, aplastic anemia, and adverse drug reactions. In ITP, an abnormal autoantibody, usually immunoglobulin $\mathrm{G}$ binds to circulating platelet membranes. Hemorrhages are the only clinical manifestations and they may be mild, such as petechiae, easy bruising, epistaxis, and gingival bleeding, although severe complications such as hemoptysis, gastrointestinal and genitourinary tract bleeding are also observed and are generally associated with marked thrombocytopenia. The most frequent cause of death in association with ITP is intracranial bleeding $[10,11]$. There was no adrenal hemorrhage reported in patients with ITP. We present a unique patient with ITP and adrenal hematoma.

Imaging studies are important in the diagnosis of adrenal masses. Both CT and magnetic resonance imaging (MRI) were successfully used to diagnose adrenal hemorrhage. Ac-

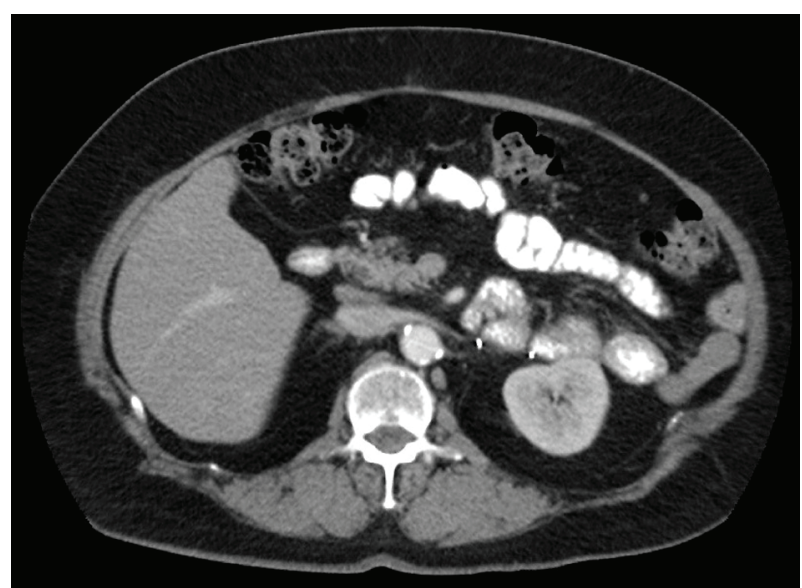

Figure 3. Abdominal CT with contrast 2 years after surgery, axial view. No evidence of recurrence.

cording to the literature, adrenal hematomas usually appeared as rounded or oval hypodense heterogeneous masses with or without calcifications, and absence of contrast enhancement. Benign adrenal lesions bigger than $6 \mathrm{~cm}$ are extremely rare and always should be surgically excised, after evaluation for possible secreting adrenal tumor [2, 3]. Adrenal hematomas could masquerade malignant neoplasm like in our patient, who had 7 $\times 9 \times 6 \mathrm{~cm}$ adrenal mass, enhancing with IV contrast.

Hormonal testing to distinguish functional and nonfunctional adrenal mass is extremely important and should include evaluation for pheochromocytoma, Cushing's disease, primary and secondary hyperaldosteronism, and virilizing tumors. All biochemical studies in our patient were negative for hormoneproducing neoplasm.

Adrenal hematomas were previously described in patients with bleeding diathesis, such as heparin-induced thrombocytopenia and anticoagulation therapy; however, there were no reports in patients with ITP. This is a first case of adrenal mass simulating neoplasm in patient with ITP.

\section{Conclusion}

Adrenal hematomas are rare and could simulate malignancy. This is a first report of adrenal hematoma in ITP, presenting as a mass. Lesions larger than $6 \mathrm{~cm}$ should be surgically excised after ruling out hormonally producing tumor.

\section{Competing Interests}

The author declares that he does not have any competing interests.

\section{Abbreviations}

CT: computer tomography; MRI: magnetic resonance imaging; ITP: idiopathic thrombocytopenic purpura; DHEAS: de- 
hydroepiandrosterone; NSAIDs: non-steroidal anti-inflammatory drugs; LUQ: left upper quadrant

\section{References}

1. Greendyke RM. Adrenal Hemorrhage. Am J Clin Pathol. 1965;43:210-215.

2. Christoforides C, Petrou A, Loizou M. Idiopathic unilateral adrenal haemorrhage and adrenal mass: a case report and review of the literature. Case Rep Surg. 2013;2013:567186.

3. Fedala NS, Haddam AM, Chentli F, Youcef RS, Kalafat N. Adrenal Hematoma: A Report of Three Cases. Journal of endocrinology and diabetes mellitus. 2014;2:117-121

4. Vella A, Nippoldt TB, Morris JC, 3rd. Adrenal hemorrhage: a 25-year experience at the Mayo Clinic. Mayo Clin Proc. 2001;76(2):161-168.

5. Kobayashi T, Uenoyama S, Miura K, Takehara Y. Idiopathic unilateral adrenal hematoma: report of a case. Surg Today. 2004;34(3):279-282.
6. Abdullah KG, Stitzlein RN, Tallman TA. Isolated adrenal hematoma presenting as acute right upper quadrant pain. J Emerg Med. 2012;43(4):e215-217.

7. Imachi H, Murao K, Yoshimoto T, Sugimoto M, Kakehi Y, Hayashi T, Kushida Y, et al. Idiopathic unilateral adrenal hemorrhage in an elderly patient. Endocrine. 2010;37(2):249-252.

8. el Khader K, el Ghorfi MH, Ouali M, Ibnattya A, Hachimi M, Lakrissa A. [Spontaneous hematoma of the adrenal glands]. Prog Urol. 2001;11(3):517-519.

9. Marti JL, Millet J, Sosa JA, Roman SA, Carling T, Udelsman R. Spontaneous adrenal hemorrhage with associated masses: etiology and management in 6 cases and a review of 133 reported cases. World J Surg. 2012;36(1):75-82.

10. Kessler CM, Sandler SG. Immune Thrombocytopenic Purpura- Medscape Reference. April 21, 2014.

11. Cortelazzo S, Finazzi G, Buelli M, Molteni A, Viero P, Barbui T. High risk of severe bleeding in aged patients with chronic idiopathic thrombocytopenic purpura. Blood. 1991;77(1):31-33. 